\title{
PURE SUBGROUPS OF TORSION-FREE GROUPS
}

\author{
PAIIL HIII, AND CHARIES MEGIBBEN
}

\begin{abstract}
In this paper, we show that certain new notions of purity stronger than the classical concept are relevant to the study of torsion-free abelian groups. In particular, implications of *-purity, a concept introduced in one of our recent papers, are investigated. We settle an open question (posed by Nongxa) by proving that the union of an ascending countable sequence of *-pure subgroups is completely decomposable provided the subgroups are. This result is false for ordinary purity. The principal result of the paper, however, deals with $\Sigma$-purity, a concept stronger than *-purity but weaker than the usual notion of strong purity. Our main theorem. which has a number of corollaries including the recent result of Nongxa that strongly pure subgroups of separable groups are again separable, states that a $\Sigma$-pure subgroup of a $k$-group is itself a $k$-group.

Among other results is the negative resolution of the conjecture (valid in the countable case) that a strongly pure subgroup of a completely decomposable group is again completely decomposable.
\end{abstract}

1. Introduction. Using the concept of type, R. Baer [B] classified completely decomposable, torsion-free groups in 1937. (All groups considered are abelian.) The fact that direct summands of completely decomposable groups are again completely decomposable is known as the Baer-Kulikov-Kaplansky Theorem and was proved for the general case only in 1958 by Kaplansky [K]. Consideration of completely decomposable groups leads naturally to what Baer called a separable group. The torsion-free group $G$ is said to be separable (in the sense of Baer) if each finite subset of $G$ is contained in a completely decomposable direct summand of $G$. Pure subgroups of completely decomposable groups are much more general than direct summands. They need not be completely decomposable, and their structure has been determined only in very special cases; see, for example, $[\mathbf{R}]$. In the finite rank case, pure subgroups of completely decomposable groups are generally known as Butler groups and have been studied at length; see, for example, [Bu, $\mathbf{A}$, and $\mathbf{A V}$ ].

In this paper, we are primarily interested in groups of infinite rank. Our main results are for a concept of purity stronger than the classical concept, but yet general enough to be widely applicable. Indeed, this new concept of purity seems most relevant to the modern theory of torsion-free and mixed groups. For example, the recent results in $[\mathbf{N}]$ on strongly pure subgroups become a part of our more general

Received by the editors November 25, 1986. Presented to the Society in San Antonio, January 1987. 1980 Mathematics Subject Classification (1985 Revision). Primary 20K20; Secondary 20K27.

The authors were supported in part, respectively, by NSF grants DMS-8521770 and DMS-8420900. 
and, perhaps, more natural theory. Not only do our results reach beyond those in [N], but we are able to answer specific questions left open in that paper.

Most of the different versions of purity studied in the literature stem from a homological base, but our new notion of purity is arithmetic in nature.

2. Variances of purity. The classical notion of a subgroup $H$ being pure in the abelian group $G$ is that $n G \cap H=n H$ for each positive integer $n$. If $G$ is torsion-free, this is equivalent to $G / H$ being again torsion-free. We will consider presently several kinds of purity, but when we say simply that $H$ is pure in $G$, the above is what we mean. We now consider three stronger versions of purity, and we show how these compare with one another.

Strong purity. A subgroup $H$ of $G$ is strongly pure in $G$ if, for any element $h \in H$, there exists a homomorphism $\phi_{h}: G \rightarrow H$ that leaves $h$ fixed. It is well known [JR] that if $H$ is strongly pure in $G$ and $S$ is a finite subset of $H$, then there exists a homomorphism $\phi_{S}: G \rightarrow H$ that leaves the elements of $S$ fixed. Thus if $H$ has finite rank and is a strongly pure subgroup of a torsion-free group $G$, then $H$ is a direct summand of $G$. This suggests a natural question, which was raised in [N] but left open. Must a strongly pure subgroup $H$ of a completely decomposable torsion-free group $G$ again be completely decomposable? It was shown in $[\mathbf{N}]$ that the answer is in the affirmative for a countable $G$, but we shall prove here that in general the answer is "no".

Star-purity. The concept of *-purity, introduced in $\left[\mathbf{H M}_{\mathbf{1}}\right]$, requires some further notation. By a height sequence $s$ we understand a sequence $\left\{s_{p}\right\}_{p \in P}$ indexed by the set $P$ of primes where each $s_{p}$ is either a nonnegative integer or the symbol $\infty$. The collection of all height sequences forms a lattice when the operations are defined in the obvious pointwise fashion. With each element $x$ of the torsion-free group $G$ we associate its height sequence $|x|$ where $|x|_{p}$ is the height of $x$ in $G$ at the prime $p$. Each height sequence $s$ then gives rise to the fully-invariant subgroup $G(s)=\{x \in$ $G:|x| \geqslant s\}$. Recall that height sequences $s$ and $t$ are said to be equivalent, $s \sim t$, if (adopting the convention $\infty-\infty=0$ ) $\sum_{p \in P}\left|s_{p}-t_{p}\right|$ is finite. A type is just an equivalence class of height sequences. We also need the fully-invariant subgroups $G\left(s^{*}\right)=\langle x \in G(s):|x|+s\rangle$ and $G\left(s^{*}, p\right)=G\left(s^{*}\right)+p(G(s))$ where $p$ is a prime. Finally, we say that a pure subgroup $H$ is *-pure in $G$ if $H \cap G\left(s^{*}\right)=H\left(s^{*}\right)$ and $H \cap G\left(s^{*}, p\right)=H\left(s^{*}, p\right)$ for all height sequences $s$ and all primes $p$. We remark that if $G$ is completely decomposable and $H$ is a *-pure subgroup of finite rank, then it is not difficult to show that $H$ is a direct summand of $G$ (see [DR]).

Sigma-purity. This is the new concept of purity that we are introducing in this paper. A subgroup $H$ of an abelian group $G$ is said to be $\Sigma$-pure provided that $h=g_{1}+g_{2}+\cdots+g_{n}$ with $h \in H$ and $g_{i} \in G\left(s_{i}\right)$ implies that $h=h_{1}+h_{2}$ $+\cdots+h_{n}$ where $h_{i} \in H\left(s_{i}\right)$. It is interesting to observe that $\Sigma$-purity agrees with ordinary purity in the local case, where only one prime is involved. Moreover, for many purposes, the concept of $\Sigma$-purity seems to be the right notion of purity for torsion-free and mixed groups. Indeed, the fact that $\Sigma$-purity is most relevant to nonlocal groups (where heights are not linearly ordered) is manifested in $\S 3$, where we show that $\Sigma$-pure subgroups inherit the property of being a $k$-group or a 
separable group. The latter result-a $\Sigma$-pure subgroup of a separable group is again separable-implies the main result of $[\mathbf{N}]$ : a strongly pure subgroup of a separable group is again separable. Obviously, all of these results are refinements of the well-known theorem by $L$. Fuchs $[\mathbf{F}]$ that asserts that a summand of a separable group is again separable.

Proposition 2.1. Strongly pure $\Rightarrow \Sigma$-pure $\Rightarrow *$-pure $\Rightarrow$ pure.

Proof. Let $H$ be strongly pure in $G$ and suppose that $h=g_{1}+g_{2}+\cdots+g_{n}$, where $h \in H$ and $g_{i} \in G\left(s_{i}\right)$. Since $H$ is strongly pure, there exists $\phi_{h}: G \rightarrow H$ that leaves $h$ fixed. Hence if $\phi_{h}\left(g_{i}\right)=h_{i}$, we have that $h=h_{1}+h_{2}+\cdots+h_{n}$, where $h_{i} \in H\left(s_{i}\right)$ since homomorphisms do not decrease heights.

Now assume that $H$ is $\Sigma$-pure but relinquish the hypothesis that $H$ is strongly pure. If $h \in H \cap G(s)$ then, by definition of $\Sigma$-purity, $h \in H(s)$ and at least $H$ is pure in $G$. Suppose now that $h \in G\left(s^{*}\right)$. Then $h=g_{1}+g_{2}+\cdots+g_{n}$ where $\left|g_{i}\right|=s_{i} \geqslant s$ and $s_{i} \times s$. Applying the $\Sigma$-purity of $H$, we obtain $h=h_{1}+h_{2}$ $+\cdots+h_{n}$ where $\left|h_{i}\right| \geqslant s_{i}$. Therefore, $h \in H\left(s^{*}\right)$. Similarly $H \cap G\left(s^{*}, p\right)=$ $H\left(s^{*}, p\right)$, so $H$ is $*$-pure in $G$. Finally, in order to be $*$-pure, $H$ is explicitly required to be pure. This completes the proof of the proposition.

REMARK. The results and examples contained herein will demonstrate that none of the implications of the preceding proposition is reversible.

3. Pure subgroups of completely decomposable groups. As promised above, we shall resolve an open question from [N] by showing that a strongly pure subgroup of a completely decomposable group, though necessarily a separable group, need not itself be completely decomposable. In order to establish this, it is convenient (perhaps essential) to have in our possession a new criterion that a pure subgroup must satisfy if it is completely decomposable. Precisely, we need a special case of a result that appears in $[\mathbf{A H}]$ and, for sake of completeness and for the convenience of the reader, we give a simplified proof here of the version we require.

THEOREM 3.1 [AH]. Let $H$ be a pure subgroup of a torsion-free group $G$. In order for $H$ to be completely decomposable it must be a separable subgroup (in the sense of Hill).

Proof. First we need to define exactly what a separable subgroup means (in the sense of Hill $\left.\left[\mathbf{H}_{1}, \mathbf{A H}\right]\right)$. A subgroup $H$ of $G$ is separable in $G$ if for each $g \in G$ there is a countable subset $\left\{h_{n}\right\}_{n<\omega}$ of $H$ satisfying the following condition: for $h \in H$, there is a corresponding $n$ such that $|g+h| \leqslant\left|g+h_{n}\right|$.

Suppose that $H$ is completely decomposable and appears in the torsion-free group $G$ as a pure subgroup. Assume that $H$ is not separable in $G$. Since $H$ is not separable, there is a $g \in G$ such that for each countable subset $C$ of $H$ there is a corresponding element $h_{C} \in H$ with the property that $|g+x| \geqslant\left|g+h_{C}\right|$ fails for each $x$ in $C$. Letting $H=\oplus_{i \in I} A_{i}$, where $A_{i}$ has rank 1 , we can choose an ascending sequence of countable subsets $J(n)$ of $I$, with $J(0)=\varnothing$, such that the following condition is satisfied where $H_{n}=\oplus_{j \in J(n)} A_{j}:$ If $h_{n} \in H_{n}$ and the inequality $\left|g+h_{n}\right|_{p} \supsetneqq|g+h|_{p}$ holds for some $h \in H$ and prime $p$, then the inequality 
already holds for some $h \in H_{n+1}$. [Here, “æ” is appropriate rather than " $<$ " due to the convention that $\infty<\infty$.]

Set $H_{\omega}=\cup_{n<\omega} H_{n}=\bigoplus_{j \in J(\omega)} A_{j}$ where $J(\omega)=\bigcup_{n<\omega} J(n)$. Since $H_{\omega}$ is countable, there exists $h \in H$ such that $|g+x| \geqslant|g+h|$ fails for every $x$ in $H_{\omega}$. In particular, $\left|g+h^{\prime}\right| \geqslant|g+h|$ fails where $h^{\prime}$ is the projection of $h$ onto $H_{\omega}$ and consequently $\left|g+h^{\prime}\right|_{p} \lesseqgtr|g+h|_{p}$ for some prime $p$. Thus, according to the preceding condition, there is already some $h^{\prime \prime}$ in $H_{\omega}$ such that $\left|g+h^{\prime}\right|_{p} \lesseqgtr\left|g+h^{\prime \prime}\right|_{p}$. It then follows that

$$
\left|h^{\prime}-h^{\prime \prime}\right|_{p}=\left|g+h^{\prime}\right|_{p}|g+h|_{p} \wedge\left|g+h^{\prime \prime}\right|_{p} \leqslant\left|h-h^{\prime \prime}\right|_{p} .
$$

But this is absurd since the choice of $h^{\prime}$ as the projection of $h$ onto $H_{\omega}$ implies that $\left|h^{\prime}-x\right|_{p} \geqslant|h-x|_{p}$ for all $x \in H_{\omega}$. We conclude, therefore, that the assumption that $H$ is not separable in $G$ must be false, and the theorem follows.

We are now prepared to answer Nongxa's question.

THEOREM 3.2. Strongly pure subgroups of completely decomposable groups need not be completely decomposable.

Proof. Denote by $S$ the set of all height sequences with no $\infty$ 's. For each $s \in S$, let $G_{s}$ be a rank 1 torsion-free group with an element $g_{s}$ whose height sequence in $G_{s}$ is exactly $s$. Set $G=\oplus_{s \in S} G_{s}$. Denote by $\phi_{s}$ the map from $G_{s}$ to the group of rationals $Q$ that takes $g_{s}$ onto 1 , and let $\phi=\sum \phi_{s}$. Observe that $\phi: G \rightarrow Q$ is epic (because all height sequences $s$ are used except those with $\infty$ 's). Let $H$ be the kernel of $\phi$ and suppose $h \in H$. Since $h \in G$, we can write, for some positive integer $m$,

$$
m h=m_{1} g_{s(1)}+m_{2} g_{s(2)}+\cdots+m_{r} g_{s(r)}
$$

where $s(1), s(2), \ldots, s(r)$ are distinct elements of $S$ and $m_{i} \in Z$. Observe that

$$
\sum_{i \leqslant r} m_{i}=\sum_{i \leqslant r} m_{i} \phi\left(g_{s(i)}\right)=\phi(m h)=0 .
$$

Now select $t \in S$ so that $t \geqslant s(i)$ and $t+s(i)$ for each $i \leqslant r$. Since $\sum_{i<r} m_{i}=0$, we have $m h=\sum_{i \leqslant r} m_{i}\left(g_{s(i)}-g_{t}\right)$ where each $g_{s(i)}-g_{t}$ is in $H$. Moreover, by Corollary 2.6 in $\left[\mathbf{H M}_{1}\right]$, we have a direct decomposition

$$
G=\left(\bigoplus_{i \leqslant r}\left\langle g_{s(i)}-g_{t}\right\rangle_{*}\right) \oplus\left(\underset{s \neq s(i)}{\bigoplus} G_{s}\right)
$$

(where, as usual, $A_{*}$ denotes the pure subgroup generated by $A$ ). Since $H_{0}=$ $\oplus_{i \leqslant r}\left\langle g_{s(i)}-g_{t}\right\rangle_{*}$ is a finite rank summand of $G$ contained in $H$ and since $h \in H_{0}$, it quickly follows that $H$ is strongly pure in $G$; indeed, $\phi_{h}$ is simply the projection of $G$ onto $H_{0}$. In order to show that $H$ is not completely decomposable, we employ Theorem 3.1. In other words, we show that $H$ is not separable in $G$.

Assume that $H$ is separable in $G$. Let $s$ denote the height sequence consisting entirely of zeroes and let $g=g_{s}$. According to the assumption that $H$ is separable in $G$, there exists a countable number of elements $h_{1}, h_{2}, \ldots, h_{n}, \ldots$ in $H$ such that if $h \in H$ there exists $n$ (depending on the choice of $h$ ) with the property that $|g+h| \leqslant\left|g+h_{n}\right|$. Observe that no nonzero element of $G$ can have an infinity in its height sequence. Since both $g$ and the $h_{n}$ 's are fixed, the height sequences $\left|g+h_{n}\right|$ 
are fixed, for each $n$, and have no $\infty$ 's. Select a height sequence $t^{\prime} \in S$ so that, at the $n$th prime, $t^{\prime}$ exceeds $\left|g+h_{n}\right|$; for example, if $p$ is the $n$th prime,

$$
t_{p}^{\prime}=\left|g+h_{n}\right|_{p}+1 \text {. }
$$

Finally, let $h^{\prime}=g_{t^{\prime}}-g=g_{t^{\prime}}-g_{s^{\prime}}$. Observe that $\left|g+h^{\prime}\right|=\left|g_{t^{\prime}}\right|$ and that this contradicts the supposition that $\left|g+h^{\prime}\right| \leqslant\left|g+h_{n}\right|$ for some $n$. Therefore, $H$ is not separable in $G$ and according to Theorem 3.1 cannot be completely decomposable.

4. $\Sigma$-pure subgroups of $k$-groups. Torsion-free $k$-groups, introduced in [ $\mathbf{H M}_{\mathbf{1}}$ ], can be defined as those torsion-free groups in which each finite subset is contained in a completely decomposable *-pure subgroup (see [DR]). But we prefer to return to the original definition in terms of primitive elements and *-valued coproducts. A nonzero element $x$ of the torsion-free group $G$ is said to be primitive if $x \notin G\left(s^{*}, p\right)$ for all $s \sim|x|$ with $s_{p}=|x|_{p} \neq \infty$. If $G$ is completely decomposable, then $x$ is primitive if and only if $\langle x\rangle_{*}$ is a direct summand (see 2.9 in [HM $]$ ). A direct sum $A=\bigoplus_{i \in I} A_{i}$ of independent subgroups of $G$ is said to be a *-valuated coproduct if $A \cap F=\bigoplus_{i \in I} A_{i} \cap F$ for all fully-invariant subgroups $F$ of the form $G(s), G\left(s^{*}\right)$, or $G\left(s^{*}, p\right)$. A pure subgroup $H$ is knice in $G$ if for each finite subset $S$ of $G$ there correspond primitive elements $x_{1}, \ldots, x_{n}$ such that $H \oplus\left\langle x_{1}\right\rangle_{*} \oplus \cdots \oplus\left\langle x_{n}\right\rangle_{*}$ is a *-valuated coproduct containing $S$. Once the preceding concepts have emerged the notion of a $k$-group is imperative, for $G$ is a $k$-group if 0 is a knice subgroup. A fundamental fact about $k$-groups that we shall use repeatedly is the following: If $H=\left\langle x_{1}\right\rangle_{*} \oplus \cdots \oplus\left\langle x_{n}\right\rangle_{*}$ is a $*$-valuated coproduct where each $x_{i}$ is a primitive element of the $k$-group $G$, then $H$ is a pure knice subgroup of $G$ (see Proposition 4.2 in $\left.\left[\mathrm{HM}_{\mathbf{1}}\right]\right)$.

If $H$ is a pure knice subgroup of $G$, then $H$ is $\Sigma$-pure in $G$. Indeed suppose $h=g_{1}+\cdots+g_{n}$ is in $H$ and let $S=\left\{g_{1}, \ldots, g_{n}\right\}$. Then, by definition of kniceness, there is a *-valuated coproduct $H \oplus A$ in $G$ that contains $S$. Writing $g_{i}=h_{i}+a_{i}$ where $h_{i} \in H$ and $a_{i} \in A$, we see that $\left|g_{i}\right| \leqslant\left|h_{i}\right|$ and $h=h_{1}+\cdots+h_{n}$. One should note, however, that kniceness is not an inductive property.

In this section, we generalize the results of $[\mathbf{N}]$ in two directions. First, our results apply to $k$-groups, not just separable groups. Second, our results are for $\Sigma$-pure subgroups, not just strongly pure subgroups. The following is a simple example that demonstrates that the generalizations are genuine in both directions.

Let $G=\left\langle\oplus_{\aleph_{0}} Z, 2 \Pi_{\aleph_{0}} Z\right\rangle$. Since $G$ is $\aleph_{1}$-free, all nonzero elements are primitive and if the direct sum $F=\left\langle x_{1}\right\rangle \oplus \cdots \oplus\left\langle x_{n}\right\rangle$ is pure in $G$, then it is a $*$-valuated coproduct. Thus $G$ is a $k$-group, but, as is well known, not a separable group [G, p. 114]. In fact the element $x=(2,2, \ldots, 2, \ldots)$ is contained in no finite rank direct summand. It follows that $H=\langle x\rangle=\langle x\rangle_{*}$ is knice in $G$ and hence $\Sigma$-pure, but $H$ is not strongly pure since it fails to be a direct summand.

We are now ready for our main theorem.

THEOREM 4.1. A $\Sigma$-pure subgroup of a torsion-free $k$-group is again a $k$-group.

Proof. Let $H$ be a $\sum$-pure subgroup of the torsion-free $k$-group $G$. Since, in particular, $H$ is *-pure in $G$, elements in $H$ are primitive if and only if they are primitive in $G$ and a direct sum of subgroups in $H$ is a *-valuated coproduct in $H$ if 
and only if it is a *-valuated coproduct in $G$. If $A=\left\langle a_{1}\right\rangle_{*} \oplus \cdots \oplus\left\langle a_{m}\right\rangle_{*}$ is a *-valuated coproduct with the $a_{i}$ 's primitive in $G$, then we shall refer to $A$ as a finite rank free *-valuated subgroup. Now let $S$ be a finite subset of $H$. To show that $H$ itself is a $k$-group, it suffices to construct a finite rank free $*$-valuated subgroup $A$ of $G$ such that $S \subseteq A \subseteq H$. Proceeding by induction on $|S|$, we may assume that all but one element of $S$, say $h$, can be imbedded in such an $A$. But since $A$ is necessarily a pure knice subgroup of $G$, we know that $h$ is contained in a *-valuated coproduct $A \oplus G_{1} \oplus G_{2} \oplus \cdots \oplus G_{n}$ with the following conditions satisfied for each $i \leqslant n$.

(a) $G_{i}$ is homogeneous of type $\tau_{i}$.

(b) $G_{i}$ is a finite rank free *-valuated subgroup of $G$.

Moreover, since $A \subseteq H$, there is no loss of generality in assuming

(c) $h \in G_{1} \oplus G_{2} \oplus \cdots \oplus G_{n}$.

Let us also label our original condition as (d).

(d) $A \oplus G_{1} \oplus G_{2} \oplus \cdots \oplus G_{n}$ is a *-valuated coproduct in $G$.

Our proof will be completed by showing that with a judicious choice of the $G_{i}$ 's, there will be a finite rank free *-valuated subgroup $B$ contained in $G_{1} \oplus G_{2}$ $\oplus \cdots \oplus G_{n}$ such that $h \in B \subseteq H$; for under these circumstances $S$ will be contained in the finite rank free *-valuated subgroup $A \oplus B \subseteq H$.

There is no reason, of course, to expect that our original choice of the $G_{i}$ 's will have this desired property. Thus we must allow ourselves the freedom to enlarge, at most finitely many times, the $G_{i}$ 's until the construction of the desired $B$ is possible. We shall, however, always adhere to the restrictions (a)-(d) and so, in particular, $n$ and the types $\tau_{1}, \tau_{2}, \ldots, \tau_{n}$ remain fixed throughout our proof. Furthermore, whenever we replace $G_{i}$ by a larger $G_{i}^{\prime}$, we insist that $G_{i}^{\prime}=G_{i} \oplus C_{i}$ be a *-valuated coproduct with $C_{i}$ a finite rank homogeneous free *-valuated subgroup of type $\tau_{i}$. The key to our being able to make such enlargements is the fact that, at any stage, the subgroup $A \oplus \dot{G}_{1} \oplus G_{2} \oplus \cdots \oplus G_{n}$ will be a pure knice subgroup of $G$. As the types $\tau_{i}$ shall not vary, we agree once and for all to arrange them in such an order that $\tau_{i}$ is minimal in the set $\left\{\tau_{i}, \tau_{i+1}, \ldots, \tau_{n}\right\}$.

The ultimate construction of $B \subseteq G_{1} \oplus G_{2} \oplus \cdots \oplus G_{n}$ depends on a secondary induction. So let $0 \leqslant r<n$ and suppose we have found elements $h_{1}, h_{2}, \ldots, h_{r}$ in $H$, together with an auxiliary subgroup $C$, such that, in conjunction with a suitable choice of the $G_{i}$ 's satisfying (a)-(d), we have the following five conditions satisfied.

(1) $C$ is a finite rank free $*$-valuated subgroup each homogeneous component of which is of type distinct from any of the $\tau_{i}$ 's.

(2) $A \oplus G_{1} \oplus G_{2} \oplus \cdots \oplus G_{n} \oplus C$ is a *-valuated coproduct in $G$.

(3) For each $i \leqslant r, h_{i} \in G_{i} \oplus G_{i+1} \oplus \cdots \oplus G_{n} \oplus C$.

(4) For each $i \leqslant r,\left|h_{i}\right|=\left|g_{i}\right|$ where $g_{i}$ is the projection of $h_{i}$ in $G_{i}$.

(5) $h-\sum_{i \leqslant r} h_{i}=y_{r+1}+y_{r+2}+\cdots+y_{n}$ where $y_{j} \in H$ and type $\left(y_{j}\right) \geqslant \tau_{j}$ for $j=r+1, r+2, \ldots, n$.

If $r=0$, it is understood that $C=0$ and that conditions (3) and (4) are vacuous. Moreover, for $r=0,(2)$ and (5) reduce to (d) and (c), respectively. Hence the induction hypotheses hold for $r=0$. 
Assuming $r<n$, we now show that these hypotheses also hold for $r+1$. By (5), we can write $h-\sum_{i \leqslant r} h_{i}=y_{r+1}+y_{r+2}+\cdots+y_{n}$ where type $\left(y_{j}\right) \geqslant \tau_{j}$. Since $A \oplus$ $G_{1} \oplus G_{2} \oplus \cdots \oplus G_{n} \oplus C$ is knice in $G$, by an appropriate enlargement of $C$ and the $G_{i}$ 's, we may suppose without loss of generality that $y_{r+1}, y_{r+2}, \ldots, y_{n}$ are actually contained in this subgroup. Since $h-\sum_{i \leqslant r} h_{i}$ is in $G_{1} \oplus G_{2} \oplus \cdots \oplus G_{n} \oplus C$ and since the conditions type $\left(y_{j}\right) \geqslant \tau_{j}$ preclude any of the elements $y_{r+1}, \ldots, y_{n}$ from having nonzero projections in $G_{1}, \ldots, G_{r}$, we may write

(i) $h-\sum_{i \leqslant r} h_{i}=g_{r+1}+g_{r+2}+\cdots+g_{n}+c$,

where $g_{j} \in G_{j}$ for $j=r+1, r+2, \ldots, n$ and $c \in C$. Furthermore, considering the projections into $C$ of the individual $y_{j}$ 's, we see that $c=d_{r+1}+d_{r+2}+\cdots+d_{n}$ where type $\left(d_{j}\right)>\tau_{j}$ for each $j$. Next, utilizing the fact that $H$ is $\Sigma$-pure in $G$, we write

(ii) $h-\sum_{i \leqslant r} h_{i}=x_{r+1}+x_{r+2}+\cdots+x_{n}+w_{r+1}+w_{r+2}+\cdots+w_{n}$ where the $x_{j}$ 's and $w_{j}$ 's are in $H$ with $\left|g_{j}\right| \leqslant\left|x_{j}\right|$ and $\left|d_{j}\right| \leqslant\left|w_{j}\right|$ for $j=r+1, r+2, \ldots, n$. Once again, enlarging $C$ and the $G_{i}$ 's, we may assume that the $x_{j}$ 's and $w_{j}$ 's are also contained in $A \oplus G_{1} \oplus G_{2} \oplus \cdots \oplus G_{n} \oplus C$. In fact, since $A \subseteq H$, all the above conditions are satisfied if we replace the $x_{j}$ 's and $w_{j}$ 's by their projections in $G_{1} \oplus G_{2} \oplus \cdots \oplus G_{n} \oplus C$. Moreover, because of the arrangement of the $\tau_{i}$ 's and the conditions on the types of the $x_{j}$ 's and $w_{j}$ 's, these elements must in fact lie in $G_{r+1} \oplus G_{r+2} \oplus \cdots \oplus G_{n} \oplus C$ and, among them all, only $x_{r+1}$ can have nonzero projection into $G_{r+1}$. It follows, comparing equations (i) and (ii), that $g_{r+1}$ is the projection of $x_{r+1}$ into $G_{r+1}$ and, consequently, that $\left|x_{r+1}\right|=\left|g_{r+1}\right|$.

Now set $h_{r+1}=x_{r+1}$ and observe that the only thing keeping $h_{1}, h_{2}, \ldots, h_{r}, h_{r+1}$ from satisfying the five conditions of the secondary induction hypotheses is the unfortunate appearance of the term $w_{r+1}$ in equation (ii). Nevertheless, it is clear that we can write

$$
h-\sum_{i \leqslant r+1} h_{i}=g_{r+2}^{\prime}+\cdots+g_{n}^{\prime}+c_{1}+c_{2}+\cdots+c_{r+1}
$$

where $g_{j}^{\prime} \in G_{j}$ for $j=r+2, \ldots, n$ and $c_{i}$ is the projection of $-h_{i}$ into $C$ for $i=1,2, \ldots, r+1$. Therefore $\left|h_{i}\right| \leqslant\left|c_{i}\right|$ and, because of conditions (1), $c_{i} \in G\left(s_{i} *\right)$ $\subseteq G\left(\tau_{i}^{*}\right)$ where $s_{i}=\left|h_{i}\right|$. Once again we apply the fact that $H$ is $\Sigma$-pure in $G$ and write

$$
h-\sum_{i \leqslant r+1} h_{i}=y_{r+2}^{\prime}+\cdots+y_{n}^{\prime}+z_{1}+z_{2}+\cdots+z_{r+1}
$$

where the $y_{j}^{\prime}$ 's and $z_{i}$ 's are in $H$ with $\left|g_{j}^{\prime}\right| \leqslant\left|y_{j}^{\prime}\right|$ for $j=r+2, \ldots, n$, and $z_{i} \in$ $G\left(s_{i} *\right) \subseteq G\left(\tau_{i}^{*}\right)$ for $i=1,2, \ldots, r+1$. As before, enlarging $C$ and the $G_{i}$ 's, we may assume that the $y_{j}^{\prime}$ 's and $z_{i}$ 's are in $G_{1} \oplus G_{2} \oplus \cdots \oplus G_{n} \oplus C$. Notice furthermore that $z_{i} \in G\left(\tau_{i}^{*}\right)$ implies that $z_{i} \in G_{i+1} \oplus G_{i+2} \oplus \cdots \oplus G_{n} \oplus C$. Finally, we let $h_{i}^{\prime}=h_{i}-z_{i}$ for $i=1,2, \ldots, r+1$ and observe that the projection of $h_{i}^{\prime}$ in $G_{i}$ is still $g_{i}$. Moreover, by $2.3,2.5$ and 2.7 of $\left[\mathbf{H M}_{1}\right],\left|h_{i}^{\prime}\right|=\left|h_{i}\right|=\left|g_{i}\right|$. Hence $h_{1}^{\prime}, h_{2}^{\prime}, \ldots, h_{r+1}^{\prime}$ satisfy all five conditions of the secondary induction.

Having completed the secondary induction, we conclude that $h=h_{1}+h_{2}$ $+\cdots+h_{n}$ with $h_{i} \in H \cap\left(G_{i} \oplus G_{i+1} \oplus \cdots \oplus G_{n} \oplus C\right)$ and $\left|h_{i}\right|=\left|g_{i}\right|$ where $g_{i}$ is the projection of $h_{i}$ in $G_{i}$. Then, by 2.5 and 2.7 of $\left[\mathbf{H} \mathbf{M}_{1}\right]$, the $h_{i}$ 's are primitive 
elements (or else 0 ) and a succession of applications of $2.5\left[\mathbf{H M}_{\mathbf{1}}\right]$ lead to the desired conclusion that $B=\left\langle h_{1}\right\rangle_{*} \oplus\left\langle h_{2}\right\rangle_{*} \oplus \cdots \oplus\left\langle h_{n}\right\rangle_{*}$ is a *-valuated coproduct. What is more, $A \oplus\left\langle h_{1}\right\rangle_{*} \oplus\left\langle h_{2}\right\rangle_{*} \oplus \cdots \oplus\left\langle h_{n}\right\rangle_{*}$ is a $*$-valuated coproduct in $H$ containing $S$, and the proof of Theorem 4.1 is complete.

COROLlary 4.2. A strongly pure subgroup of a k-group is again a k-group.

COROllary $4.3[\mathbf{N}]$. A strongly pure subgroup of a separable group is again a separable group.

Proof. By Proposition 2.1 and Theorem 4.1, a strongly pure subgroup $H$ of a separable group $G$ is at least a $k$-group. To show that $H$ is separable it suffices by Theorem 4.3 of $\left[\mathbf{H M}_{1}\right]$ to argue that any pure and knice subgroup $A$ of $H$ having finite rank is a direct summand of $H$. But, in fact, $A$ is a direct summand of $G$.

COROLlaRY 4.4. A $\Sigma$-pure subgroup of a separable group is strongly pure.

COROllary $4.5\left[\mathbf{H M}_{1}, \mathbf{F}\right]$. A direct summand of a k-group or a direct summand of a separable group is again, respectively, a k-group or separable.

COROllary 4.6 [HM, DR]. A pure knice subgroup of a k-group is again a k-group.

We close this section with one further general observation concerning $\Sigma$-pure subgroups of $k$-groups.

Proposition 4.7. If $G$ is a $k$-group, then every infinite subset can be imbedded in a $\Sigma$-pure subgroup of the same cardinality.

Proof. Since $\Sigma$-purity is an inductive property, it suffices to show that an arbitrary countable subset $S$ of the $k$-group $G$ can be imbedded in a countable $\sum$-pure subgroup. Let $S=\cup_{n<\omega} S_{n}$ where each $S_{n}$ is finite. We need only prove that there exists an ascending sequence $\left\{H_{n}\right\}_{n<\omega}$ of finite rank pure knice subgroups of $G$ such that $S_{n} \subseteq H_{n}$ for each $n<\omega$; for then $H=\bigcup_{n<\omega} H_{n}$ will be a countable $\Sigma$-pure subgroup containing $S$. The existence of a finite rank pure knice subgroup $H_{0} \supseteq S_{0}$ follows immediately from Proposition 4.2 in $\left[\mathbf{H M}_{\mathbf{1}}\right]$ and the fact that $G$ is a $k$-group. Then given $H_{0}, \ldots, H_{n}$ with the desired properties, the kniceness of $H_{n}$, implies that there are primitive elements $x_{1}, \ldots, x_{m}$ such that $H_{n+1}=H_{n} \oplus\left\langle x_{1}\right\rangle_{*}$ $\oplus \cdots \oplus\left\langle x_{m}\right\rangle_{*}$ is a $*$-valuated coproduct containing $S_{n+1}$. But again using 4.2 of [HM $\mathbf{M}_{1}$, we conclude that $H_{n+1}$ is also a pure knice subgroup of $G$. Thus, the desired sequence $\left\{H_{n}\right\}_{n<\omega}$ exists by induction, and the proof is complete.

COROllaRy 4.8 [DR]. An uncountable k-group is the smooth union of $k$-groups of smaller cardinality.

5. Unions of *-pure subgroups. Another question raised in $[\mathbf{N}]$ but left open is whether or not a torsion-free group which is the ascending union of a sequence of strongly pure completely decomposable subgroups is itself completely decomposable. An affirmative answer is given below under the weaker assumption that the subgroups are *-pure. Recall that $G$ is said to be an fo-union of the family of 
subsets $\left\{G_{\alpha}\right\}_{\alpha \in A}$ if for each finite subset $S$ of $G$ there exists an $\alpha$ such that $S \subseteq G_{\alpha}$. In particular, an ascending union is an fo-union. This section is devoted to the proof and some consequences of the following theorem.

THEOREM 5.1. If the torsion free group $G$ is the fo-union of a sequence $\left\{G_{n}\right\}_{n<\omega}$ of *-pure completely decomposable subgroups, then $G$ is completely decomposable.

To place this result in the same context as the analogous theorems established in $\left[\mathbf{H}_{2}\right]$ for totally projective groups and in $\left[\mathbf{H M}_{2}\right]$ for $p$-local Warfield groups, we shall base its proof on the Axiom 3 characterization of completely decomposable groups obtained in $\left[\mathbf{H M}_{\mathbf{1}}\right]$.

Definition 5.2. A family $\mathscr{C}$ of subgroups of $G$ is said to be an Axiom 3 family if the following three conditions are satisfied:

(i) $0 \in \mathscr{C}$.

(ii) If $A_{i} \in \mathscr{C}$ for each $i \in I$, then $\sum_{i \in I} A_{i} \in \mathscr{C}$.

(iii) If $A \in \mathscr{C}$ and $S$ is a countable subset of $G$, then there is a $B \in \mathscr{C}$ such that $\langle S, A\rangle \subseteq B$ and $B / A$ is countable.

In Theorem 5.3 of $\left[\mathbf{H M}_{\mathbf{1}}\right]$, we show that a torsion-free group $G$ is completely decomposable if and only if there is an Axiom 3 family $\mathscr{C}$ consisting of pure knice subgroups of $G$. Indeed, as the proof of that theorem demonstrates, it suffices to exhibit a family $\mathscr{C}$ of pure knice subgroups satisfying (i), (iii) and the weaker condition

(ii') $\mathscr{C}$ is closed under the union of chains.

In broad outline, we shall prove Theorem 5.1 by selecting, for each $n<\omega$, an Axiom 3 family $\mathscr{C}_{n}$ of pure knice subgroups of $G_{n}$ and then utilize all these $\mathscr{C}_{n}$ 's to extract from $G$ a family $\mathscr{C}$ of pure knice subgroups satisfying (i), (ii') and (iii). In fact, the description of $\mathscr{C}$ is quite simple. We take $\mathscr{C}$ to consist of all subgroups $A$ of $G$ which satisfy the following two conditions:

(1) $A$ is a pure subgroup of $G$ such that $A \cap G_{n} \in \mathscr{C}_{n}$ for all $n<\omega$.

(2) For each $n<\omega,\left(A+G_{n}\right) \cap F=(A \cap F)+\left(G_{n} \cap F\right)$ for all fully invariant subgroups $F$ of the form $G(s), G\left(s^{*}\right)$ or $G\left(s^{*}, p\right)$ where $s$ is any height sequence and $p$ is an arbitrary prime.

Notice that (2) is clearly an inductive property and, in view of (ii), so is (1). Thus it is evident that $\mathscr{C}$ satisfies (i) and (ii').

It is also easy to prove that each $A \in \mathscr{C}$ is a knice subgroup of $G$. Indeed let $S$ be a finite subset of $G$ and choose $n<\omega$ such that $S \subseteq G_{n}$. By (1), there exist primitive elements $x_{1}, \ldots, x_{m}$ in $G_{n}$ and a *-valuated coproduct $N=\left(A \cap G_{n}\right) \oplus\left\langle x_{1}\right\rangle$ $\oplus \cdots \oplus\left\langle x_{m}\right\rangle$ which contains $S$. Because of the *-purity of $G_{n}$, this is actually a *-valuated coproduct in $G$ and, moreover, the $x_{i}$ 's are primitive in $G$. Taking $C=\left\langle x_{1}, \ldots, x_{m}\right\rangle$, it suffices to argue that $N^{\prime}=A \oplus C$ is a *-valuated coproduct in $G$, that is, we need to show that $(A \oplus C) \cap F=(A \cap F) \oplus(C \cap F)$ for all $F$ of the form $G(s), G\left(s^{*}\right)$ or $G\left(s^{*}, p\right)$. Let us suppose then that $a+c \in F$ where $a \in A, c \in C$ and $F$ is any such fully invariant subgroup. By (2), $a+c=a_{1}+x$ where $a_{1} \in A \cap F$ and $x \in G_{n} \cap F$. Then $a-a_{1}=x-c$ is an element of $A \cap G_{n}$ such that $\left(a-a_{1}\right)+c=x$ is in $\left(\left(A \cap G_{n}\right) \oplus C\right) \cap F$. But since $N=\left(A \cap G_{n}\right) \oplus C$ 
is a *-valuated coproduct in the *-pure subgroup $G_{n}$ and since $a_{1} \in F$, it follows that $a \in A \cap F$ and $c \in C \cap F$, as desired.

All that remains to complete the proof of Theorem 5.1 is to verify that $\mathscr{C}$ satisfies the countable extension property (iii). Toward this end, we require a lemma which quite likely will prove useful in future applications. In keeping with the terminology and notation of $\left[\mathbf{H}_{\mathbf{1}}\right]$, we say that the subgroups $A$ and $H$ are compatible and write $A \| H$ provided for each pair $(a, h) \in A \times H$ there exists an $h^{\prime} \in A \cap H$ such that $\left|a+h^{\prime}\right| \geqslant|a+h|$. As our usage suggests, compatibility is a symmetric relation and, in fact, it is readily checked that $A \| H$ for subgroups of the torsion-free group $G$ if and only if $(A+H) \cap G(s)=(A \cap G(s))+(H \cap G(s))$ for all height sequences $s$.

LEMMA 5.3. If $S$ is a countable subset of the torsion-free group $G$ and if the subgroup $H$ is separable (in the sense of Hill), then there is a countable pure subgroup $A$ of $G$ such that $A \supseteq S$ and $(A+H) \cap F=(A \cap F)+(H \cap F)$ for all fully invariant subgroups $F$ of the form $G(s), G\left(s^{*}\right)$ or $G\left(s^{*}, p\right)$.

Proof. First we show that we can at least find a countable pure subgroup $A \supseteq S$ such that $A \| H$. Actually it suffices to construct an ascending sequence $\left\{A_{n}\right\}_{n<\omega}$ of countable pure subgroups such that $A_{0}=\langle S\rangle_{*}$ and, for each $n<\omega$, if $(a, h) \in A_{n}$ $\times H$, then there is an $h^{\prime} \in A_{n+1} \cap H$ such that $\left|a+h^{\prime}\right| \geqslant|a+h|$; for then $A=$ $\cup_{n<\omega} A_{n}$ will be compatible with $H$. Suppose then that $A_{0}, \ldots, A_{n}$ having the desired properties have been constructed and let $a_{1}, a_{2}, \ldots, a_{m}, \ldots$ be an enumeration of the elements of $A_{n}$. Since $H$ is separable in $G$, we can associated with each $a_{m} \in A_{n}$ a corresponding sequence $\left\{h_{m, i}\right\}_{i<\omega}$ in $H$ with the following property: If $h \in H$, then $\left|a_{m}+h\right| \leqslant\left|a_{m}+h_{m, i}\right|$ for some $i<\omega$. Now take $A_{n+1}$ to be the pure closure of the subgroup of $G$ generated by $A_{n}$ and the doubly infinite collection of all the $h_{m, i}$ 's. It is clear that $A_{n+1}$ satisfies the requisite condition and hence the construction of the desired sequence $\left\{A_{n}\right\}_{n<\omega}$ follows by induction.

Next we show that given a countable subgroup $A$ of $G$ with $A \| H$, we can find a countable subgroup $B \supseteq A$ such that $(A+H) \cap F \subseteq(B \cap F)+H$ for all fully invariant subgroups $F$ of the form $G(s), G\left(s^{*}\right)$ or $G\left(s^{*}, p\right)$. For notational convenience, let $G\left(s^{*}, 0\right)=G\left(s^{*}\right)$ and take $\mathscr{F}$ to consist of all fully invariant subgroups $F$ of the above forms for which $(A+H) \cap F \nsubseteq(A \cap F)+H$. Since $A \| H$, each $F \in \mathscr{F}$ is of the form $G\left(s^{*}, p\right)$ for some height sequence $s$ and some $p$ in $\mathbf{P} \cup\{0\}$. Now for each $F=G\left(s^{*}, p\right)$ in $\mathscr{F}$, let $A_{F}$ consist of all elements $a \in A \cap G(s)$ such that $|a| \sim s$ and $(a+H) \cap F \nsubseteq(A \cap F)+H$. We shall first show that $A_{F} \neq \varnothing$ for each $F \in \mathscr{F}$, which (because of the countability of $A, \mathbf{P}$ and any given type $\sigma)$ will imply that $\mathscr{F}$ is countable. Indeed suppose $F=G\left(s^{*}, p\right)$ is in $\mathscr{F}$ and we have $a \in A$ and $h \in H$ such that $a+h$ is in $F$ but not in $(A \cap F)+H$. Since $A \| H$, we may assume that $a \in A \cap G(s)$. But then it follows that $|a| \sim s$, since otherwise we would have $a+h \in\left(A \cap G\left(s^{*}\right)\right)+H \subseteq(A \cap F)$ $+H$. Thus $A_{F} \neq \varnothing$, as claimed. Now for each $F \in \mathscr{F}$ and each $a \in A_{F}$, fix some $h_{a} \in H$ such that $a+h_{a} \in F$ and $h_{a} \notin F$. Since $\mathscr{F}$ and each $A_{F}$ is countable, the definition of the $G\left(s^{*}, p\right)$ 's implies that there is a countable subgroup $B \supseteq A$ such 
that $a+h_{a} \in B \cap F$ for all $F \in \mathscr{F}$ and all $a \in A_{F}$. It is then trivial that $(A+H)$ $\cap F \subseteq(B \cap F)+H$ for all fully invariant $F$ of the form $G(s), G\left(s^{*}\right)$ or $G\left(s^{*}, p\right)$.

Finally, to obtain a countable subgroup $A$ satisfying our lemma, we apply the constructions of the last two paragraphs to build up a nested sequence $\langle S\rangle \subseteq A_{1} \subseteq$ $B_{1} \subseteq \cdots \subseteq A_{n} \subseteq B_{n} \subseteq \cdots$ of countable subgroups such that, for each $n, A_{n}$ is pure in $G, A_{n} \| H$ and $\left(A_{n}+H\right) \cap F \subseteq\left(B_{n} \cap F\right)+H$ for all fully invariant subgroups $F$ of the form $G(s), G\left(s^{*}\right)$ or $G\left(s^{*}, p\right)$. Then the subgroup $A=\cup_{n=1}^{\infty} A_{n}=$ $\bigcup_{n=1}^{\infty} B_{n}$ obviously has the desired property, and the proof of the lemma is complete.

We are now in position to finish the proof of Theorem 5.1 by showing that our $\mathscr{C}$ satisfies (iii) of Definition 5.2. So let $A$ be a fixed element of $\mathscr{C}$ and observe that each quotient group $G_{n} / A \cap G_{n}$ is completely decomposable since

$$
\mathscr{C}_{n}^{\prime}=\left\{C / A \cap G_{n}: C \in \mathscr{C}_{n} \text { and } C \supseteq A \cap G_{n}\right\}
$$

is an Axiom 3 family of pure knice subgroups. Also since $A$ is pure in $G$ and $A \| G_{n}$, it follows that $A+G_{n}$ is pure in $G$. Thus, by Theorem 3.1, each of the completely decomposable groups $A+G_{n} / A$ is separable (in the sense of Hill) in the group $G / A$. For the moment, let us fix $n<\omega$ and take $H=G_{n}$. Since $A$ is a pure knice subgroup of $G$, it is balanced in $G$ by Theorem 4.3 in [HM $\mathbf{M}_{1}$. Consequently, $(G / A)(s)=G(s)+A / A$ for all height sequences $s$ and, of course, similar relations hold for $(G / A)\left(s^{*}\right)$ and $(G / A)\left(s^{*}, p\right)$. Because $A+H / A=A+G_{n} / A$ is separable in $G / A$, Lemma 5.3 allows us to select, for any given countable subset $S$ of $G$, a countable subgroup $B_{1} / A$ of $G / A$ with $B_{1} \supseteq\langle S, A\rangle$ and such that

$$
\left(B_{1} / A+(A+H) / A\right) \cap(F+A / A)=\left(\left(B_{1} \cap F\right)+A / A\right)+((H \cap F)+A / A)
$$

for all fully invariant subgroups $F$ of the form $G(s), G\left(s^{*}\right)$ or $G\left(s^{*}, p\right)$. But since $A$ satisfies (2) and $H=G_{n}$, it follows readily that $\left(B_{1}+H\right) \cap F=\left(B_{1} \cap F\right)+$ $\left(H \cap F\right.$ ) for all such $F$ 's. Then, for our fixed $n$, choose $C_{1} \in \mathscr{C}_{n}$ such that $C_{1} \supseteq B_{1} \cap G_{n}$ and $C_{1} / A \cap G_{n}$ is countable. (This is possible because $\mathscr{C}_{n}$ satisfies (iii) and $B_{1} / A$ is countable.) Continuing in this manner, we construct two ascending sequences of subgroups $\left\{B_{m}\right\}_{m=1}^{\infty}$ and $\left\{C_{m}\right\}_{m=1}^{\infty}$ such that, for all $m, B_{m} / A$ and $C_{m} / A \cap G_{n}$ are countable, $B_{m} \cap G_{n} \subseteq C_{m} \subseteq B_{m+1}, C_{m} \in \mathscr{C}_{n}$ and $\left(B_{m}+H\right) \cap F=\left(B_{m} \cap F\right)+(H \cap F)$ for all fully-invariant subgroups $F$ of the form $G(s), G\left(s^{*}\right)$ or $G\left(s^{*}, p\right)$. If we now take $B$ to be the union of the $B_{m}$ 's and $C$ the union of the $C_{m}$ 's, we will have $B \cap G_{n}=C$ and $B / A$ countable. Moreover, since $\mathscr{C}_{n}$ satisfies (ii), the subgroup $B$ will, for our fixed choice of $n$, satisfy the conditions formulated in (1) and (2). Finally, by using a sequence running infinitely often through $\omega$ and exploiting the inductive nature of the various conditions under consideration, we can find such a $B$ satisfying (1) and (2) for all $n<\omega$. Thus, $\mathscr{C}$ does indeed satisfy condition (iii), and the proof of Theorem 5.1 is complete.

Since *-purity reduces to ordinary purity in homogeneous groups, we have the following immediate consequence of our theorem.

COROLlaRY 5.4 [S]. If the homogeneous torsion-free group $G$ is the fo-union of a countable sequence of completely decomposable pure subgroups, then $G$ is completely decomposable. 
Let $m$ be an infinite cardinal. Recall that a torsion-free group $G$ is said to be $m$-separable if every subset of cardinality less than $m$ is contained in a completely decomposable direct summand of $G$.

COROllary 5.5. If cof $m=\omega$, then any $m$-separable group of cardinality $m$ is completely decomposable.

That *-purity in the hypotheses of Theorem 5.1 cannot be weakened to ordinary purity is confirmed by the following example.

EXample 5.6 [S]. Consider the direct sum $A=\oplus_{i<\omega} A_{i}$ where $A_{0}=\left\langle a_{0}\right\rangle$ and, for $i \geqslant 1, A_{i}=\left\langle a_{i}\right\rangle_{*}$ with $\left|a_{i}\right|=(\infty, 0,0, \ldots, 0, \ldots)$. Let $p_{0}, p_{1}, p_{2}, \ldots, p_{i}, \ldots$ be the increasing sequence of primes and introduce elements $x_{1}, x_{2}, \ldots, x_{i}, \ldots$ such that $p_{i} x_{i}=a_{0}+a_{i}$ for each $i \geqslant 1$. Then the group $G=\left\langle A, x_{1}, x_{2}, \ldots, x_{i}, \ldots\right\rangle$ is the ascending union of the subgroups $G_{n}=\left\langle\oplus_{i \leqslant n} A_{i}, x_{1}, x_{2}, \ldots, x_{n}\right\rangle$. A routine computations shows that the $G_{n}$ 's are pure in $G$. Moreover, each $G_{n}$ is completely decomposable since

$$
G_{n}=\left\langle b_{n}\right\rangle \oplus A_{1} \oplus \cdots \oplus A_{n}
$$

where $b_{n}=\lambda_{1}^{(n)} x_{1}+\lambda_{2}^{(n)} x_{2}+\cdots+\lambda_{n}^{(n)} x_{n}$ and the integers $\lambda_{i}^{(n)}$ are chosen so that $1=\sum_{i=1}^{n} \lambda_{i}^{(n)}\left(p_{1} p_{2} \cdots p_{n} / p_{i}\right)$. Let $\tau$ denote the type determined by $(\infty, 0,0, \ldots, 0, \ldots)$ and observe because of the purity and form of the $G_{n}$ 's that $G(\tau)=\oplus_{i \geqslant 1} A_{i}$. Now if $G$ were to be completely decomposable, then we would have a direct decomposition $G=B \oplus C(\tau)$ where $B$ is necessarily a rank one group. But since $G$ is the ascending union of the pure subgroups $G_{n}$, the form of the $G_{n}$ 's forces us to the conclusion that $B$ must be cyclic. This, however, is absurd since the nonzero element $a_{0}+G(\tau)$ is divisible in $G / G(\tau)$ by each of the primes $p_{1}, p_{2}, \ldots, p_{i}, \ldots$

6. A deficiency of *-purity. In this section, we show that our main result cannot be carried over to *-purity. In other words, a $*$-pure subgroup of a $k$-group need not be a $k$-group, nor must a $*$-pure subgroup of a separable group again be separable. In fact, we will construct an example of a *-pure subgroup of a countable completely decomposable group that is not itself completely decomposable. Indeed, the subgroup we construct will have no nonzero primitive elements at all! This demonstrates that $*$-purity is much weaker than $\Sigma$-purity.

The following proposition is well known; at least, it is a consequence of elementary set theory.

Proposition 6.1. Let $\mathscr{F}$ denote the (countable) collection of all finite subsets of the set $P$ of primes. Let $\left\{F_{n}\right\}_{n<\omega}$ be a sequence such that (1) $F_{n} \in \mathscr{F}$, and (2) for each $F \in \mathscr{F}$, there are infinitely many $n$ for which $F_{n}=F$. There exists a sequence $\left\{E_{n}\right\}_{n<\omega}$ that satisfies the following conditions whenever $i, j<\omega$.

(1) $E_{i} \subseteq P$.

(2) $E_{i}$ is infinite and $E_{i} \supseteq F_{i}$.

(3) $E_{i} \cap E_{j}$ is finite when $i \neq j$.

(4) $E_{k} \cap E_{j}$ properly contains $E_{i} \cap E_{j}$ when $i \neq j$ and $k>\max \{i, j\}$. 
We are now ready to construct the example referred to above and prove the following.

THEOREM 6.2. A countable *-pure subgroup of a completely decomposable group need not be completely decomposable. Indeed, such a subgroup need not possess primitive elements.

Proof. Select the sequence $\left\{E_{n}\right\}_{n<\omega}$ so that it satisfies the conditions (1)-(4) of the preceding proposition. Let $s_{n}$ be the idempotent height sequence (consisting of 0 's and $\infty$ 's) such that $s_{n}$ assumes the value $\infty$ at $p$ if and only if $p \in E_{n}$. Choose a rank one group $G_{n} \subseteq Q$ so that the height sequence of $g_{n}$ in $G_{n}$ is precisely $s_{n}$. Let $G=\oplus_{n<\omega} G_{n}$. Let $\phi_{n}$ be the map from $G_{n}$ to $Q$ that maps $g_{n}$ onto 1 ; and let $\phi$ : $G \rightarrow Q$ be the induced map, $\phi=\sum \phi_{n}$. Finally, denote the kernel of $\phi$ by $H$.

Observe that $H=\operatorname{ker} \phi$ is pure in $G$ since $\phi$ maps $G$ into the torsion-free group $Q$. We claim that $H$ is *-pure in $G$. In order to show $H$ is *-pure in $G$, we need to verify

(i) $H \cap G(s)=H(s)$,

(ii) $H \cap G\left(s^{*}\right)=H\left(s^{*}\right)$, and

(iii) $H \cap G\left(s^{*}, p\right)=H\left(s^{*}, p\right)$.

We know already that condition (i) is satisfied since $H$ is pure in $G$. We intend to verify that if $x \in H$ and $x \neq 0$, then the following holds.

(iv) $x \in H\left(|x|^{*}\right)$.

This will not only yield immediately conditions (ii) and (iii), but in addition it will preclude $x$ from being primitive.

Suppose $x \in H$ and $x \neq 0$. For some positive integer $m$, we can write (by virtue of $x \in G$ )

$$
m x=m_{1} g_{1}+m_{2} g_{2}+\cdots+m_{r} g_{r},
$$

where $m_{i} \in Z$. Recall that $g_{i} \in G_{i}$ has height exactly $s_{i}$, where $s_{i}$ is idempotent and $s_{i}(p)=\infty$ if and only if $p \in E_{i}$. There is no loss of generality in assuming that $m=1$; for if $p \mid m$ and $p+m_{i}$, then $s_{i}(p)=\infty$. Therefore, $p g_{i}^{\prime}=g_{i}$ where $g_{i}^{\prime}$ in $G_{i}$ has exactly the same height sequence as $g_{i}$. Thus by replacing $g_{i}$ by $g_{i}^{\prime}$, if necessary, we may assume that $p \mid m_{i}$ for each $i$ in which case $p$ can be cancelled from both $m$ and the $m_{i}$ 's. Consequently, we assume that $x=m_{1} g_{1}+m_{2} g_{2}+\cdots+m_{r} g_{r}$. Since $x$, in fact, belongs to $H$, we know that $\sum_{i<r} m_{i}=\phi(x)=0$. In particular, we know that at least two $m_{i}$ 's are different from zero since $x \neq 0$.

Observe that if we let

$$
s=\left|m_{1} g_{1}\right| \wedge\left|m_{2} g_{2}\right| \wedge \cdots \wedge\left|m_{r} g_{r}\right|
$$

then $|x|=s$. Consequently, $|x|$ has only a finite number of $\infty$ 's. More to the point, by invoking condition (4) of Proposition 6.1, we conclude that there exists $k>r$ such that $E_{k} \cap E_{i}$ properly contains $E_{j} \cap E_{i}$ whenever $i, j \leqslant r$ and $i \neq j$. Moreover,

$$
x=m_{1}\left(g_{2}-g_{k}\right)+m_{2}\left(g_{2}-g_{k}\right)+\cdots+m_{r}\left(g_{r}-g_{k}\right)
$$


because $\sum_{i \leqslant r} m_{i}=0$. But $\left|m_{i}\left(g_{i}-g_{k}\right)\right| \geqslant s$ and $\left|m_{i}\left(g_{i}-g_{k}\right)\right|$ has at least one more $\infty$ than $s$ (whether computed in $H$ or $G$ since $H$ is pure). This implies that $x \in H\left(s^{*}\right)$. Since $s=|x|$, we have verified condition (iv), and the theorem is proved.

\section{REFERENCES}

[AH] U. Albrecht and P. Hill, Butler groups of finite rank and axiom 3 (to appear).

[A] D. Arnold, Pure subgroups of finite rank completely decomposable groups, Lecture Notes in Math., vol. 874, Springer-Verlag, New York, 1981, pp. 1-31.

[AV] D. Arnold and C. Vinsonhaler, Pure subgroups of finite rank completely decomposable groups. II, Lecture Notes in Math., vol. 1006, Springer-Verlag, New York, 1983, pp. 97-143.

[B] R. Baer, Abelian groups without elements of finite order, Duke Math. J. 3 (1937), 68-122.

[Bu] M. Butler, A class of torsion-free abelian groups of finite rank, Proc. London Math. Soc. 15 (1965), 680-698.

[DR] M. Dugas and K. Rangaswamy, On torsion-free abelian k-groups, Proc. Amer. Math. Soc. 99 (1987), 403-408.

[F] L. Fuchs, Summands of separable abelian groups, Bull. London Math. Soc. 2 (1970), 205-208.

[G] P. Griffith, Infinite abelian groups, Univ. of Chicago Press, Chicago, Ill., 1970.

$\left[\mathbf{H}_{1}\right] \mathrm{P}$. Hill, Criteria for freeness in groups and valuated vector spaces, Lecture Notes in Math., vol. 616, Springer-Verlag, New York, 1977, pp. 140-157.

$\left[\mathbf{H}_{2}\right]$ _ Criteria for total projectivity, Canad. J. Math. 23 (1981), 817-825.

$\left[\mathbf{H M}_{1}\right]$ P. Hill and C. Megibben, Torsion free groups, Trans. Amer. Math. Soc. 295 (1986), 735-751.

$\left[\mathrm{HM}_{2}\right] \ldots$ _ Axiom 3 modules, Trans. Amer. Math. Soc. 295 (1986), 715-734.

[JR] S. Janakiraman and K. Rangaswamy, Strongly pure subgroups of abelian groups, Lecture Notes in Math., vol. 574, Springer-Verlag, New York, 1977, pp. 57-64.

[K] I. Kaplansky, Projective modules, Ann. of Math. 68 (1958), 372-377.

[N] L. Nongxa, Strongly pure subgroups of separable torsion-free abelian groups, Trans. Amer. Math. Soc. 290 (1985), 363-373.

[R] R. Richman, An extension of the theory of completely decomposable torsion-free abelian groups, Trans. Amer. Math. Soc. 279 (1983), 175-185.

[S] A. Shiflet, Almost completely decomposable groups, Dissertation, Vanderbilt Univ., 1976.

Department of Mathematics, Baylor University, Waco, Texas 76798

Department of Mathematics, Vanderbilt University, Nashville, Tennessee 37235 